\title{
PREVALÊNCIA DE SOBREPESO E OBESIDADE EM ESCOLARES DO ENSINO FUNDAMENTAL DE ESCOLAS PÚBLICAS DE LIMEIRA, SÃO PAULO
}

Homero Gustavo Ferrari

Murilo Henrique Barbosa

\section{Resumo}

Objetivo: verificar a prevalência de sobrepeso e obesidade em escolares de seis a onze anos, de ambos os sexos de escolas públicas de Limeira, São Paulo. Métodos: 365 escolares (177 meninas e 188 meninos) foram submetidos a medidas antropométricas de massa corporal, estatura e índice de massa corpórea. Para a classificação de sobrepeso e obesidade foram utilizados os pontos de corte de Cole et al. (2000). Resultados: os resultados indicam que a prevalência de sobrepeso e obesidade na faixa dos 6 aos 8 anos é semelhante entre os sexos, entretanto dos 9 aos 11 é maior nas meninas.

\section{Palavras-chave}

Sobrepeso; Obesidade; Escolares.

\section{PREVALENCE OF OVERWEIGHT AND OBESITY IN PERTAINING TO SCHOOL OF THE BASIC EDUCATION OF PUBLIC SCHOOLS OF LIMEIRA, SÃO PAULO}

Homero Gustavo Ferrari

Murilo Henrique Barbosa

\begin{abstract}
Objective: to verify the prevalence of overweight and obesity in six to eleven year old schoolchildren of both sexes of public school of Limeira, São Paulo. Methods: 365 schoolchildren (177 girls and 188 boys) were submitted to anthropometrical measurements of body mass, stature and body mass index. For classification of overweight and obesity were utilized the cuttofs of Cole et al (2000). Results: the results indicated that the prevalence of overweight and obesity is similar between 6 to 8 year old in both sexes, therefore in 9 to 11 year old is great in the girls.
\end{abstract}

\section{Key-Words}

Overweight; Obesity; Schoolar. 


\section{INTRODUÇÃO}

A obesidade vem sendo considerada como uma epidemia mundial e se tornando um grave problema de saúde pública (WHO, 1990). O excesso de gordura corporal em adultos está intimamente ligado a vários fatores de risco para doenças do coração como diabetes, hipertensão arterial, hiperlipidemia, além de outros tipos de doenças crônicas (FRANCISCHI et al., 2000).

Entretanto, a obesidade não está presente somente em adultos, pelo contrário, a obesidade em jovens (crianças e adolescentes) vem aumentando muito rapidamente em alguns países do mundo incluindo o Brasil, sendo que um dos países mais afetados com a obesidade infantil sem dúvida é os Estados Unidos. Um estudo conduzido por Flegal et al.,(2001) nos Estados Unidos, verificou que a prevalência de sobrepeso em jovens de 6 a 14 anos praticamente dobrou nos últimos 30 anos, tanto em meninos como em meninas, enquanto que a prevalência da obesidade mais que dobrou nesse mesmo período. Atualmente estima-se que uma em cada cinco crianças esteja com sobrepeso ou obesidade nos Estados Unidos (BARLOW; DIETZ, 1998).

No Brasil apesar da incidência de sobrepeso e obesidade em jovens ser menor do que nos Estados Unidos em valores relativos, a proporção do aumento nos últimos 30 anos e bem parecida. Isso é demonstrado por um estudo de Wang, Monteiro e Popkin (2002), que compararam dois estudos realizados no Brasil entre os anos de 1974-1975 e 1996-1997 com jovens de 6 a 18 anos de ambos os sexos e de áreas rurais e urbanas. Os resultados revelaram que nesse período o aumento de sobrepeso e obesidade combinados, saltou de 2,9\% para $13,1 \%$ entre os jovens do sexo masculino e de 5,3\% para $14,8 \%$ entre os jovens do sexo feminino. Esse estudo também revelou que os maiores aumentos ocorreram entre os jovens das áreas urbanas de 4,9\% para 18,4\% versus rural de 3,1\% para 8,4\%.

O sobrepeso e a obesidade em jovens, assim como em adultos, também está ligada a muitos fatores de risco para doenças do coração, entre eles hipertensão arterial, diabetes, hiperlipidemia, além de complicações ortopédicas, hepática e respiratória (BARLOW; DIETZ, 1998).

Dessa forma, fica claro que o problema da obesidade infantil deve ser tratado com muita seriedade pelos órgãos competentes em todas as esferas de governo, com ações efetivas a fim de tentar frear essa epidemia. Entretanto, para que sejam traçadas estratégias e ações de prevenção e combate à obesidade infantil, é necessário conhecer como ela se desenvolve nessa população e quais suas principais causas, para isso os estudos sobre prevalência de sobrepeso e obesidade em jovens se torna importante. Assim os 
objetivos desse estudo foram verificar a prevalência de sobrepeso e obesidade em escolares do ensino fundamental de escolas públicas da cidade de Limeira e comparar a incidência de sobrepeso e obesidade entre os gêneros em diferentes faixas etárias.

\section{METODOLOGIA}

\section{Amostra}

Foram avaliadas 365 crianças na faixa de 5 a 10 anos de idade, sendo 177 do gênero feminino e 188 do gênero masculino. As crianças eram estudantes da $V$ a $4^{\text {a }}$ séries do ensino fundamental (ciclo I) de duas diferentes escolas públicas da cidade de Limeira. O presente trabalho foi aprovado pelo Comitê de Ética em Pesquisa das Faculdades Integradas Einstein de Limeira sob n 07-7/004.

\section{AVALIAÇÃO ANTROPOMÉTRICA}

Foram medidas as seguintes variáveis de crescimento: massa corporal $(\mathrm{Kg})$ e estatura $(\mathrm{cm})$, posteriormente foi calculado outro indicador o IMC $\left(\mathrm{kg} / \mathrm{m}^{2}\right)$. Para a medida de peso foi utilizada uma balança portátil digital da marca Plena ${ }^{\circledR}$ modelo Aqua 2000 com precisão de $50 \mathrm{~g}$, enquanto que para a medida da estatura foi utilizada uma régua antropométrica com precisão de $0,1 \mathrm{~mm}$ fixada na parede a 50 $\mathrm{cm}$ do solo, juntamente com um esquadro de madeira. Todas as medidas foram realizadas em duplicata adotando-se a média como valor para cálculo.

\section{PROCEDIMENTOS ADOTADOS PARA AS MEDIDAS ANTROPOMÉTRICAS}

Massa corporal: as crianças foram medidas descalças e trajando o mínimo de roupas possível na posição em pé de frente para a escala de medida da balança, com afastamento lateral dos pés, ereto e com olhar fixo à frente.

Estatura: as crianças foram medidas descalças, em posição ortostática com os pés unidos, pocurando colocar em contato com a régua as superfícies posteriores do calcanhar, cintura pélvica, cintura escapular e região occiptal. A medida foi com o esquadro em ângulo de 90 graus em relação a escala de medida e com a cabeça do avaliado orientada no plano de Frankfurt, paralela ao solo.

\section{CLASSIFICAÇÃO DE SOBREPESO E OBESIDADE}

Para a classificação de sobrepeso e obesidade foi utilizada a tabela proposta por Cole et al. (2000), para crianças e adolescentes de 2 a 18 anos de idade. Nessa classificação proposta o sobrepeso é relativo ao IMC 25,0 para adultos e a obesidade relativo ao IMC 30,0 para adultos. 


\section{ANÁLISE ESTATÍSTICA}

A apresentação dos resultados foi feita pela análise descritiva, através de média, desvio padrão e porcentagem. Para a comparação das freqüências associadas de sobrepeso e obesidade foi utilizado o teste Qui-quadrado, adotando-se como nível de significância $\mathrm{p}<0,05$.

\section{RESULTADOS}

Os dados apresentados pela tabela 1em relação aos meninos, indicam que a prevalência de sobrepeso e obesidade na faixa dos seis aos oito anos são parecidas com pequena diferença percentual, entretanto na faixa dos nove aos onze anos os índices de sobrepeso mais que dobram, enquanto os de obesidade tem uma pequena redução.

Tabela 1- Prevalência de sobrepeso e obesidade nos escolares avaliados do gênero masculino.

\begin{tabular}{ccccc}
\hline Fase escolar & Idade (anos) & $\mathbf{N}$ & $\begin{array}{c}\text { Sobrepeso } \\
(\mathbf{\%})\end{array}$ & Obesidade (\%) \\
\hline $1^{\mathrm{a}}$ série e $2^{\mathrm{a}}$ série & $\begin{array}{c}6 \text { anos e } 11 \text { meses à } 8 \\
\text { anos e 9 meses }\end{array}$ & 60 & 6,6 & 5,0 \\
$3^{\mathrm{a}}$ série e $4^{\mathrm{a}}$ série & $\begin{array}{l}9 \text { anos à } 11 \text { anos e 3 } \\
\text { meses }\end{array}$ & 128 & 15,6 & 3,9
\end{tabular}

Já entre as meninas a tabela 2 indica que na faixa dos seis aos oito anos os índices de sobrepeso são bem maiores do que de obesidade. Na faixa dos nove aos onze anos os índices de sobrepeso assim como nos meninos também chegam a dobrar, e diferentemente dos meninos os índices de obesidade também aumentam.

Tabela 2- Prevalência de sobrepeso e obesidade nos escolares avaliados do gênero feminino.

\begin{tabular}{ccccc}
\hline Fase escolar & Idade (Anos & N & Sobrepeso & Obesidade \\
\hline & 6 anos e 11 meses a & & & \\
$1^{\text {a }}$ série e $2^{\text {a }}$ Série & 8 anos e 9 meses & 62 & 9,7 & 3,2 \\
& & & & \\
$3^{\text {a }}$ série e 4 a série & 9 anos à 11 anos e 3 meses & 115 & 19,1 & 7,8
\end{tabular}

O gráfico 1 mostra os índices de sobrepeso e obesidade associados entre os gêneros nas quatro fases escolares avaliadas. Os índices de sobrepeso e obesidade associados podem dar uma visão melhor de comparação entre as faixas etárias avaliadas bem como entre os gêneros. 
Dessa forma, observamos que aos sete anos não foram encontradas diferenças significativas entre meninos e meninas nos índices de sobrepeso e obesidade associados, por outro lado diferenças significativas foram encontradas em todas as outras faixas etárias.

Foi verificado também, que por volta dos oito anos de idade os meninos tem índices de sobrepeso e obesidade maiores que as meninas $(25 \%$ x $12,5 \%)$, sendo que esse quadro passa a se inverter por volta dos nove anos .

\section{Gráfico 1- Comparação dos valores associados de sobrepeso e obesidade entre os gêneros nas quatro} fases escolares avaliadas. $(\#=\mathbf{p}<\mathbf{0 , 0 5})$

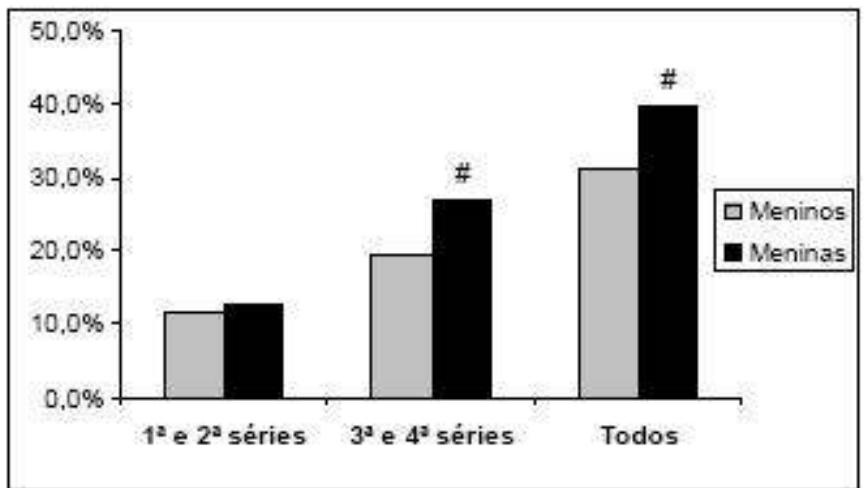

\section{DISCUSSÃO}

A discussão de estudos envolvendo o tema da obesidade em jovens é sempre muito difícil, pois a comparação entre os estudos sejam nacionais ou internacionais deve ser feita com cautela, levando-se em consideração vários fatores, como número de indivíduos avaliados, região do país ou do mundo, nível sócio-econômico, etnia, hábitos alimentares, entre outros, e principalmente os critérios utilizados para a classificação de sobrepeso e obesidade.

Atualmente a utilização do IMC para a classificação de sobrepeso e obesidade em crianças e adolescentes é um consenso na literatura específica, mas o que ainda não é um consenso são os pontos de corte utilizados para classificar sobrepeso e obesidade. Os pontos de corte utilizados nesse estudo, propostos por Cole et al. (2000) vem sendo utilizados em diversos estudos nacionais (GARCIA et al., 2004; GIUGLIANO et al., 2004; SOAR et al., 2004) e internacionais (WANG, MONTEIRO e POPKIN, 2002; CHINN e RONA, 2007), sendo considerado menos arbitrário que outros pontos de corte para estudos internacionais (FLEGAL et al., 2001). 
Em relação aos resultados desse estudo verificamos que na faixa dos seis aos oito anos tanto em meninos como em meninas os índices de sobrepeso são maiores do que os de obesidade e os índices de sobrepeso e obesidade associados são parecidos entre os gêneros, esse comportamento também tem sido apontado por outros estudos. Em um deles Sotelo, Colugnati e Taddei (2004) avaliaram a prevalência de sobrepeso e obesidade em 2.519 escolares de $V$ a $4^{\text {a }}$ série da cidade de São Paulo, nesse estudo também foram utilizados os pontos de corte propostos por Cole et al. (2000). Os autores também encontraram índices de sobrepeso maiores do que de obesidade em meninos e meninas dos seis aos oito anos, sendo $11,2 \%$ e $8 \%$ para os meninos e $12,9 \%$ e $6,1 \%$ para as meninas, entretanto, os índices associados de sobrepeso e obesidade foram parecidos entre meninos e meninas, 19,3\% e 21,4\% respectivamente. Em relação a faixa dos nove aos onze anos, é interessante observar que os autores encontraram uma diminuição dos índices de sobrepeso e obesidade entre os meninos, e diminuição dos índices de obesidade nas meninas. Esses achados corroboram com o presente estudo onde também observamos uma diminuição dos níveis de obesidade entre os meninos da mesma faixa etária, entretanto não verificamos o mesmo comportamento entre as meninas.

Com relação aos níveis de sobrepeso e obesidade associados em todas as crianças das duas faixas etárias, os maiores índices foram encontrados nas meninas em relação aos meninos, 31,1\% e 39,8\%, respectivamente.

Giugliano e Carneiro (2004) observaram em crianças de seis a dez anos do centro-oeste do Brasil, utilizando os pontos de corte sugeridos por Cole et al. (2000), valores de sobrepeso e obesidade associados de 21,1\% para os meninos e de 22,9\% para as meninas. Apesar desse comportamento ser o mesmo do presente estudo, com maiores índices para as meninas, os valores percentuais são bem menores. Outros estudos porém, tem encontrado comportamentos inversos, ou seja, com maiores índices de sobrepeso e obesidade associados para meninos. Ronque et al. (2005), por exemplo, avaliaram 511 escolares de sete a dez anos estudantes da $V$ a $4^{\text {a }}$ séries de escolas particulares da região sul do Brasil e encontraram valores de sobrepeso e obesidade associados maiores para os meninos $(37,2 \%)$ em relação as meninas $(26,6 \%)$.

Entretanto, é necessário lembrar que nesse estudo os autores adotaram como pontos de corte para sobrepeso os percentil 85 e para obesidade o percentil 95, das curvas de crescimento da NCHS (2000). Parece portanto, que os diferentes índices encontrados pelos diferentes estudos aqui apresentados, podem ter sido influenciados pelos critérios adotados como pontos de corte, região do país avaliada,

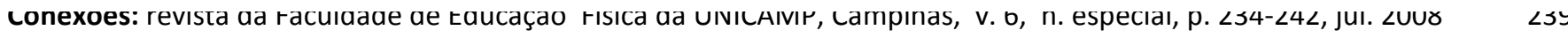
ISSN: $1983-9030$ 
número de indivíduos avaliados, além do nível sócio- econômico.

Dessa forma, concluímos que os valores de sobrepeso e obesidade entre escolares de seis a oito anos são semelhantes entre meninos e meninas, em contrapartida, na faixa dos nove aos onze anos os índices são significativamente maiores para as meninas.

Concluímos também, que na transição da faixa etária dos seis aos oito anos para a faixa etária dos nove aos onze anos, as prevalências de sobrepeso aumentam bruscamente chegando a dobrar seus valores, tanto em meninos quanto em meninas.

\section{REFERÊNCIAS}

BARLOW S. E, DIETZ WH. Obesity evaluation and treatment: expert committee recommendations. Pediatrics, v. 102, n. 3, p.1- 11, 1998.

CHINN S, RONA RJ. Prevalence and trends in overweigth and obesity in three cross sectional studies of british children, 1974-94. BMJ, v. 322, p. 24-26, 2001.

COLE TJ, BELLIZZI MC, FLEGAL KM, DIETZ WH. Establishing a standard definition for child overweight and obesity worldwide: international survey. BMJ 2000; 320:1240-44.

FLEGAL K. M, et al. Prevalence of overweigth in US children: comparison of US growth charts from the Centers for disease Control and Prevention with other reference values for body mass index. Am J Clin Nutr, v. 73, p. 1086-1093, 2001.

FRANCISCHI R. P.P. et al. Obesidade: atualização sobre sua etiologia, morbidade e tratamento. Rev Nutr, v. 13, n. 1, p. 17-28, 2000.

GARCIA F. D. et al. Avaliação de fatores de risco associados com elevação da pressão arterial em crianças. J Pediat, v. 80, n. 1, p. 29-34, 2004.

GIUGLIANO R, CARNEIRO E. C. Fatores associados à obesidade em escolares. J Pediatr, v. 80, n. 1, p. 17-22, 2004.

GUigliano, R. MELO A. L. P. Diagnóstico de sobrepeso e obesidade em escolares: utilização do índice de massa corporal segundo padrão internacional. J Pediatr, v.80, n. 2, p. 129-134, 2004.

RONQUE, V. E. R. et al. Prevalência de sobrepeso e obesidade em escolares de alto nível socioeconômico em Londrina, Paraná, Brasil. Rev Nutr, v. 18, n. 6, p. 709-717, 2005.

SOTELO Y. O. M.; COLUGNATI F.A.B.; TADDEI J. A. A C. Prevalência de sobrepeso e obesidade entre escolares da rede pública segundo três critérios de diagnóstico antropométrico. Cad Saúde Pública, v. 20, n. 1, p. 233-240, 2004.

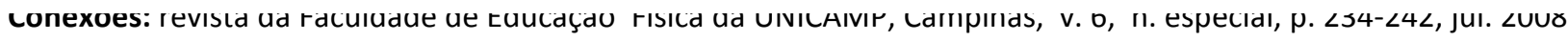
ISSN: $1983-9030$ 
SOAR, C. et al. Prevalência de sobrepeso e obesidade em escolares da uma escola pública de Florianópolis, Santa Catarina. Rev Bras Saúde Matern. Infant, v. 4, n. 4, p. 391-397, 2004.

WANG Y.; MONTEIRO C.; POPKIN B. M. Trends of obesity and underweigth in older children and adolescents in the United States, Brazil, China, and Rússia. Am J Clin Nutr, v. 75, p. 971-977, 2002 WORLD HEALTH ORGANIZATION. Diet, nutrion, and the prevention of chronic diseases. Geneva: WHO, 1990. 


\section{Homero Gustavo Ferrari}

Faculdades Integradas Einstein de Limeira - FIEL

\section{Murilo Henrique Barbosa \\ Faculdades Integradas Einstein de Limeira - FIEL}

\section{Referência do artigo:}

\section{ABNT}

FERRARI, H. G., BARBOSA, M. H. Prevalência de sobrepeso e obesidade em escolares do ensino fundamental de escolas públicas de Limeira, São Paulo. Conexões, v. 6, p. 234-242, 2008.

\section{APA}

Ferrari, H. G., \& Barbosa, M. H. (2008). Prevalência de sobrepeso e obesidade em escolares do ensino fundamental de escolas públicas de Limeira, São Paulo. Conexões, 6, 234-242.

\section{VANCOUVER}

Ferrari HG, Barbosa MH. Prevalência de sobrepeso e obesidade em escolares do ensino fundamental de escolas públicas de Limeira, São Paulo. Conexões, 2008; 6: 234-242. 